Advanced Computing: An International Journal ( ACIJ ), Vol.3, No.6, November 2012

\title{
Transmission Time and Throughput analysis of EEE LEACH, LEACH and Direct Transmission Protocol: A Simulation Based Approach
} MEENAKSHI SHARMA ${ }^{1}$ AND ANIL KUMAR. SHAW ${ }^{2}$

\author{
${ }^{1}$ Department of Computer Science and Engineering, SMIT, Majitar, Sikkim, India \\ menakshi219@gmail.com \\ ${ }^{2}$ Department of Electronics and Communication Engineering, NERIST, Nirjuli, India \\ anilshaw27@yahoo. com
}

\begin{abstract}
This paper gives a brief description about some routing protocols like EEE LEACH, LEACH and Direct Transmission protocol (DTx) in Wireless Sensor Network (WSN) and a comparison study of these protocols based on some performance matrices. Addition to this an attempt is done to calculate their transmission time and throughput. To calculate these, MATLAB environment is used. Finally, on the basis of the obtained results from the simulation, the above mentioned three protocols are compared. The comparison results show that, the EEE LEACH routing protocol has a greater transmission time than LEACH and DTX protocol and with smaller throughput.
\end{abstract}

\section{KEYWORDS}

Data, EEE LEACH, transmission time, throughput

\section{INTRODUCTION}

Wireless Sensor Networks (WSN) consists of base stations (BS) and several sensor nodes which are unattended and have limited energy resource and they can work until the energy exists inside them [1] [7]. The sensors that are attached to the nodes are capable of sensing the data from the environment in which they are deployed. The sensor nodes process data from the environment and transmit them to the BS. The BS received those data from the sensor nodes. This transmission and receiving operation is carried out by some transmitter circuits and receiving circuits which are attached with the sensor nodes and base stations respectively. The transmitter circuit uses more amount of energy as compared to the receiver circuit [1]. The power dissipated in transmission and receiving is calculated with the help of the following equations [1] [2] [6]:

Transmitting:

$\operatorname{ETX}(\mathrm{k}, \mathrm{d})=\operatorname{ETX}-\left\{\left(\mathrm{E}_{\text {elec }} * \mathrm{k}\right)+\left(\mathrm{E}_{\mathrm{mp}} * \mathrm{k} * \mathrm{~d}\right)\right\}$

$\operatorname{ETX}(\mathrm{k}, \mathrm{d})=\operatorname{ETX}-\left\{\left(\mathrm{E}_{\text {elec }} * \mathrm{k}\right)+\left(\mathrm{E}_{\mathrm{fs}} * \mathrm{k} * \mathrm{~d}\right)\right\}$

Receiving:

$\mathrm{ERX}(\mathrm{k})=\mathrm{ERX}-\left(\mathrm{E}_{\mathrm{elec}}+\mathrm{EDA}\right) * \mathrm{k}$

Where,

$\mathrm{E}_{\text {elec }}$ denotes amount of Energy consumption per bit in the transmitter or receiver circuitry.

$\mathrm{E}_{\mathrm{mp}}$ Amount of energy consumption for multipath fading.

$\mathrm{E}_{\mathrm{fs}}$ Amount of energy consumption for free space.

EDA Data aggregation energy.

DOI : $10.5121 /$ acij.2012.3609 
There are several routing protocols exist in WSN and among them the hierarchical routing protocols are the simplest, easiest and lots of research works are going on them. The wellknown Hierarchical routing protocols in WSN are LEACH, PEGASIS, TEEN, APTEEN etc. and among them LEACH is the first and simplest hierarchical routing protocol [9] [10]. Several modified versions of LEACH are proposed and among these all we are mainly concentrate on a recently developed modified version of LEACH i.e. EEE LEACH protocol [2]. In this paper we are trying to compare EEE LEACH protocol with LEACH and Direct Transmission protocol in terms of their transmission time and throughput.

Transmission time is defined as the complete time taken to transmit data packet from source to destination [5]. Throughput is defined as the number of packets transmitted over the network per unit time period [5].

The section II consists of the brief description about the three routing protocols and their comparison study. Section III represents the implementation details. In section IV simulation results along with comparisons are discussed and in section $\mathrm{V}$ we conclude the paper.

\section{Brief Description About The Protocols}

\subsection{Direct Transmission Protocols (DTx)}

This is the simplest routing protocol which consists of only BS and normal sensor nodes. The sensor nodes act as senders and BS serves as the destination node to all the other sensor nodes in the network. The sensor nodes transmit their data to the BS and the BS received these all. The communication between the sensor nodes and BS is direct without any intermediate communicator. The nodes only remain active during the data transmission to the base station. Consequently, won't spend energy on receiving the messages from the other nodes, but they will only spend the minimum data on listening the channel and, therefore, they will spend their battery capacity on sending messages to the base station [3]. On the other hand, when a sensor node transmits data directly to the base station, the energy loss incurred can be quite extensive depending on the location of the sensor nodes relative to the base station. As a result, the Direct Transmission protocol's complexity can be negligible and its implementation quasi-trivial, but it is the least energy efficient protocol in most cases [3].

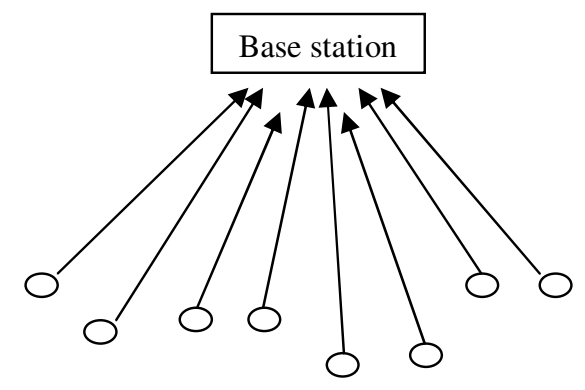

Figure 1: Architecture of Direct Transmission Protocol (DTx)

\subsection{LEACH Protocol}

LEACH stands for Low-Energy Adaptive Clustering Hierarchy. It is the first hierarchical routing protocol in Wireless Sensor network. In this protocol nodes are divided into only two types of categories; normal sensor nodes and cluster heads (CH). At first the normal sensor nodes are grouped together and form clusters and among all the sensor nodes in a cluster one node are selected as a $\mathrm{CH}$ node. The $\mathrm{CH}$ selection procedure is a random selection procedure where every node is assign a random value and this is compared with a threshold value $(T(n))$ [8][10]. If the node's random value is 
less than the $T(n)$, then the respective node can act as a $\mathrm{CH}$. The $\mathrm{T}(\mathrm{n})$ is calculated by the following formula given in eq:1 [1] [2] [4] [6]. In this way, in each and every cluster, there exists one $\mathrm{CH}$.

$$
T(n)=\left\{\begin{array}{cc}
\frac{P}{1-P *\left(r \bmod \frac{1}{P}\right)} & \text { if } \mathrm{n} \in \mathrm{G} \\
0 & \text { otherwise }
\end{array}\right.
$$

Where,

$\mathrm{n}=$ given number of nodes.

$\mathrm{p}=$ the priori probability of a node being elected as a cluster-head.

$\mathrm{r}=\mathrm{a}$ random number between 0 and 1 that is selected by a sensor node. If this random number is less than the threshold value $T(n)$, then the respective node becomes the cluster-head.

$\mathrm{G}=$ the set of nodes that were not accepted as cluster head in the last " $1 / \mathrm{p}$ " events.

At first normal sensor nodes transmit their data to their respective $\mathrm{CHs}$. On receiving these data, the $\mathrm{CHs}$ aggregated them in a compressed form and further transmit them to the BS. Finally BS received all compressed data from different $\mathrm{CHs}$ present in the network.

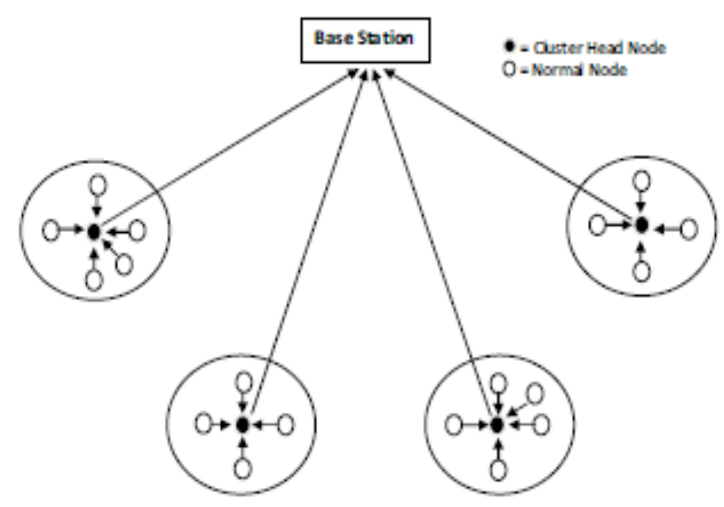

Figure 2: Architecture of LEACH Protocol [2]

\subsection{EEE LEACH Protocol}

EEE LEACH or Energy Efficient Extended LEACH [2] is an approach of multilevel clustering technique to increase energy efficiency by reducing its radio communication distance [4]. In this multilevel clustering approach besides having a single layer of clusters formation between the nodes and Base station like LEACH, it involves two layers of clusters formation [4]. In the first layer $\mathrm{CHs}$ are formed where the normal nodes transmit their own data to their respective $\mathrm{CH}$ and by using the fuse mechanism the $\mathrm{CHs}$ aggregate the received data. Again in the second layer Master Cluster Heads (MCH) are formed. After the formation of MCHs, the CHs search the nearest MCHs by calculating the distance between them and transmit their aggregate data to the respective MCHs. In the similar way, the $\mathrm{MCHs}$ received data from their nearest $\mathrm{CHs}$, aggregate all received data, transformed them into a compress format and forward them to the 
base station (BS). The number of $\mathrm{CHs}$ and $\mathrm{MCHs}$ are initially decided by using a predetermined fractional value i.e. $\mathrm{p}$ (election probability value) for $\mathrm{CHs}$ and $\mathrm{p}_{\mathrm{m}}$ (election probability value) for MCHs. In EEE LEACH, the numbers of MCHs are kept less than the number of $\mathrm{CHs}$ to minimize the overall communication distance between the nodes and Base station.

EEE LEACH protocol performs better network life-time and is more energy-efficient than LEACH protocol [2].

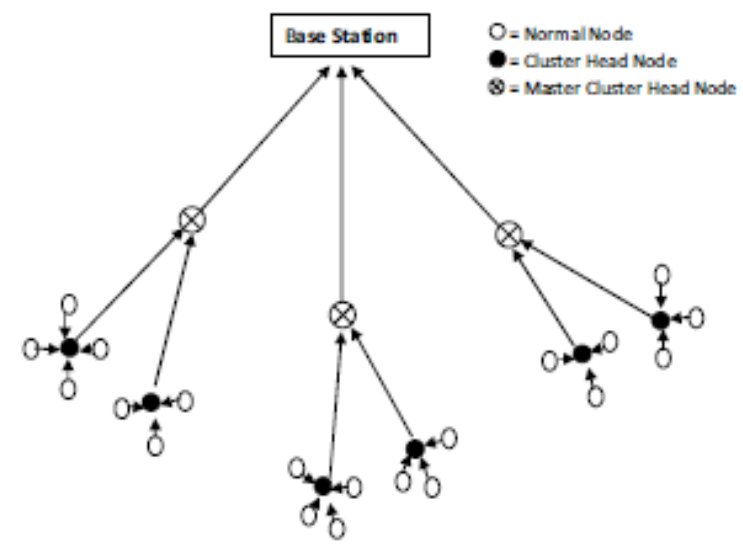

Figure 3: Architecture of EEE LEACH Protocol [2]

Comparison between DTx, LEACH and EEE LEACH protocols [8] [9] [10]:

Table 1.Comparison Table for Different Hierarchical Routing Protocols

\begin{tabular}{|l|c|c|c|}
\hline \multicolumn{1}{|c|}{ Protocol } & DTx & LEACH & EEE LEACH \\
\hline Metrics & No & Yes & Yes \\
\hline Cluster Formation & No & No & No \\
\hline Chain Formation & No & Yes & Yes \\
\hline Master Head $(\mathrm{CH})$ & No & No & Yes \\
\hline Data Aggregation & No & Yes & Yes \\
\hline Mobility & Fixed BS & Fixed BS & Fixed BS \\
\hline Scheduling & TDMA & TDMA, CDMA & TDMA, CDMA \\
\hline Network Lifetime & Average & Good & Very Good \\
\hline Energy Efficient & Least & Good & Very Good \\
\hline Communication & Single-hop & Single-hop & Multi-hop \\
\hline QoS & No & No & No \\
\hline
\end{tabular}

\subsection{Other Improved LEACH Protocols}

Energy LEACH protocol and Multihop-LEACH protocol [11] improves choice method of the cluster head and communication mode from single to multi-hop between cluster head and sink. Both have better performance than LEACH protocols. Multihop-LEACH protocols survive longer than energy-LEACH and LEACH protocol resulting in high throughput [11]. The effect of the distance between base station and centre of gravity of distribution is studied in [12]. Its affect in throughput is analysed. The closer is gives the better performance. The function of lifetime and throughput of network to the time length of each round is studied in [13]. This function can be used to enhance the lifetime and throughput of network. The overload energy problem is solved by using E-LEACH [14] over LEACH protocol. It balances the energy 
consumption of sensor nodes. The link quality between the node evaluated based on the link packet loss is shown in [15]. Considering packet loss in choosing the best communication path has a significant impact on reducing the energy consumption of the network as well as increasing network throughput [15].

\section{IMPLEMENTATIONS DETAILS}

The routing protocols DTx, LEACH and EEE LEACH have been simulated accurately in MATLAB. These have been made assuming a network having dimensions 200 x 300 meters. The number of nodes in each protocol is assumed to be 200. The cost of transmission and receiving was calculated by the formulas mentioned above in the section I. The nodes are generated and placed randomly. The parameters that are used to calculate the transmission time and throughput are given below:

- Distance between the normal nodes and Bs.

- Distance between the normal node and CHs.

- Distance between the CHs and BS.

- Distance between the CHs and MCHs.

- Distance between the MCHs and BS.

- Speed of em waves.

- Size of the data packet

\subsection{Simulation Parameter Table}

Table 2.Simulation Parameters

\begin{tabular}{|c|l|l|}
\hline \multirow{2}{*}{$\begin{array}{l}\text { Sl. } \\
\text { No. }\end{array}$} & Simulation Parameters and their Values \\
\cline { 2 - 3 } & Parameters & Value \\
\hline 1. & Routing Protocols & DTx, LEACH, EEE LEACH \\
\hline 2 & Environment Size & $200 \times 300$ \\
\hline 3 & Number of nodes & 200 \\
\hline 4 & Packet Size & $2000 \mathrm{bits}$ \\
\hline 5 & Speed of em wave & $3 \times 10^{8} \mathrm{~m} / \mathrm{s}$ \\
\hline 7 & $\begin{array}{l}\text { Election Probability value } \\
\text { of CHs (p) }\end{array}$ & $10 \%$ to $30 \%$ \\
\hline 8 & $\begin{array}{l}\text { Election Probability value } \\
\text { of MCHs (pm) }\end{array}$ & $2 \%$ to $15 \%$ \\
\hline 9 & Number of rounds & 5 to 10,000 rounds \\
\hline 10 & Initial energy per node $\left(\mathrm{E}_{0}\right)$ & $1 \mathrm{~J}$ \\
\hline 11 & $\mathrm{E}_{\text {elec }}$ & $50 \mathrm{~nJ} / \mathrm{bit}$ \\
\hline 12 & $\mathrm{E}_{\mathrm{fs}}$ & $10 \mathrm{pJ} / \mathrm{bit} / \mathrm{m}^{2}$ \\
\hline 13 & $\mathrm{E}_{\mathrm{mp}}$ & $0.0015 \mathrm{pJ} / \mathrm{bit} / \mathrm{m}^{4}$ \\
\hline 14 & $\mathrm{E}_{\mathrm{DA}}$ & $5 \mathrm{~nJ} / \mathrm{bit}$ \\
\hline
\end{tabular}

\subsection{Snapshot of the Routing Protocols}

We simulated DTx, LEACH and EEE LEACH protocols in MATLAB simulator and their simulated outputs are shown in figure 4, 5, 6 respectively. 


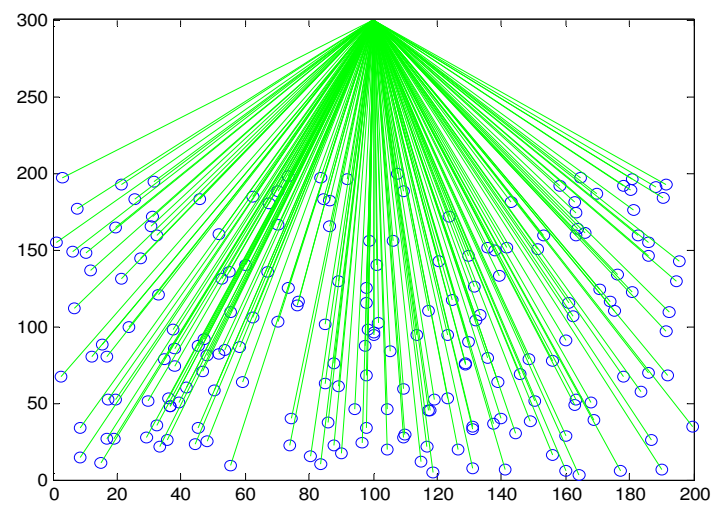

Figure 4: Direct Transmission Protocol

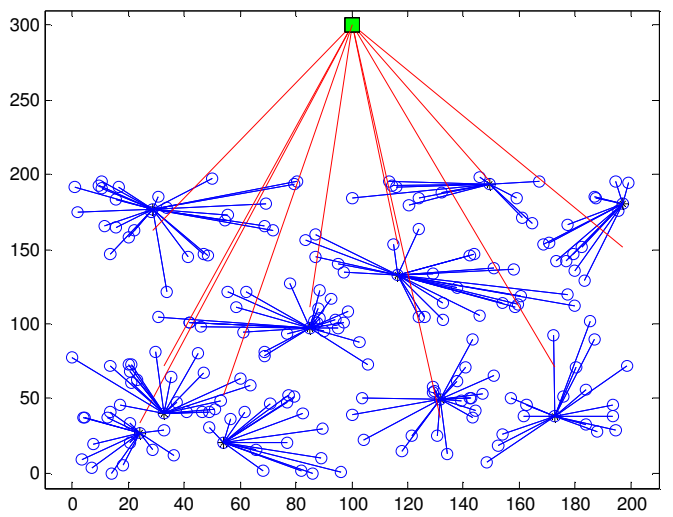

Figure 5: LEACH Protocol [2]

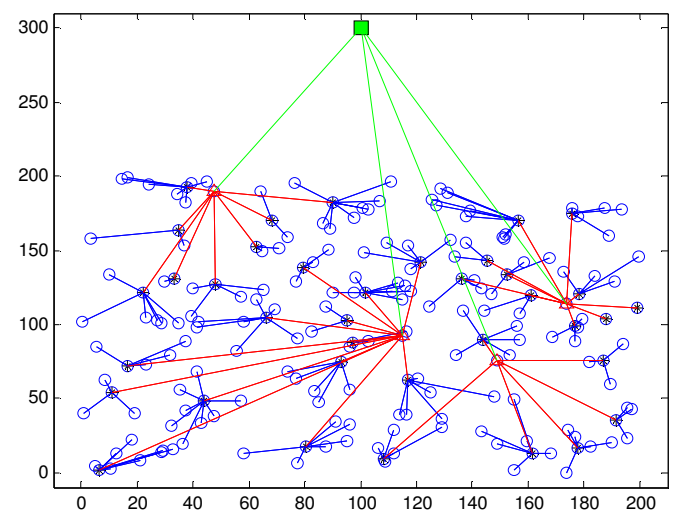

Figure 6: EEE LEACH Transmission Protocol [2]

\section{RESULTS AND DISCUSSIONS}

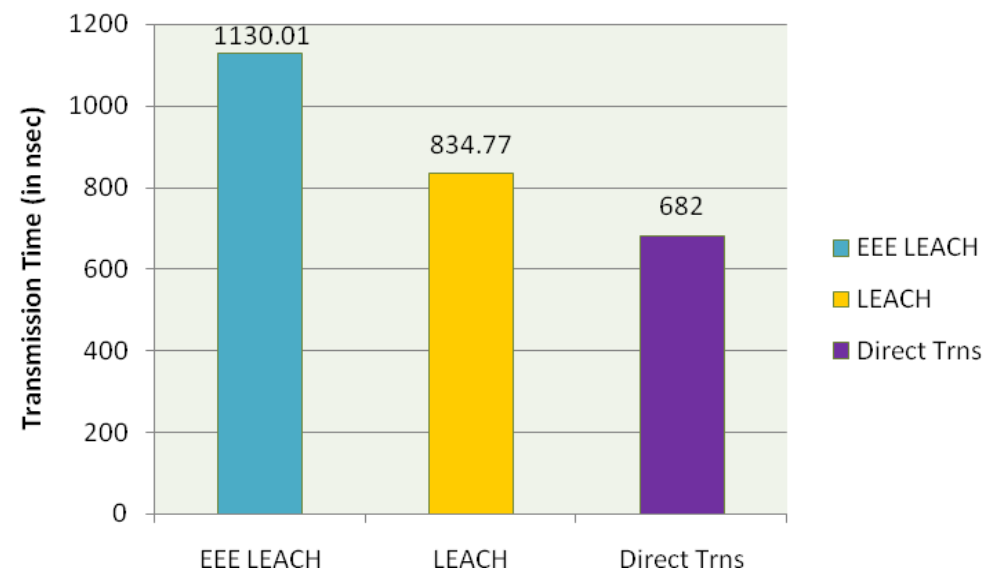

Figure 7: Transmission Time Comparison Graph between EEE LEACH, LEACH and DTx

Figure 7 shows the comparison graph of EEE LEACH, LEACH and DTx protocol in terms of their transmission time. Here we observe that after the completion of 10th round, EEE LEACH protocol takes large transmission time to transmit data to the $\mathrm{BS}$, whereas in case of $\mathrm{LEACH}$ 
and DTx protocol the transmission time is gradually decreases. The major reason behind this is that in case of EEE LEACH protocol data have to travel two layers of clustering mechanism, where in the first level they have to move from normal node to $\mathrm{CHs}$ and in second level from CHs to MCHs. But in case of LEACH, data have to travel only one level and in DTx data can directly reach to BS. Therefore EEE LEACH posses largest transmission time than LEACH and DTx protocol

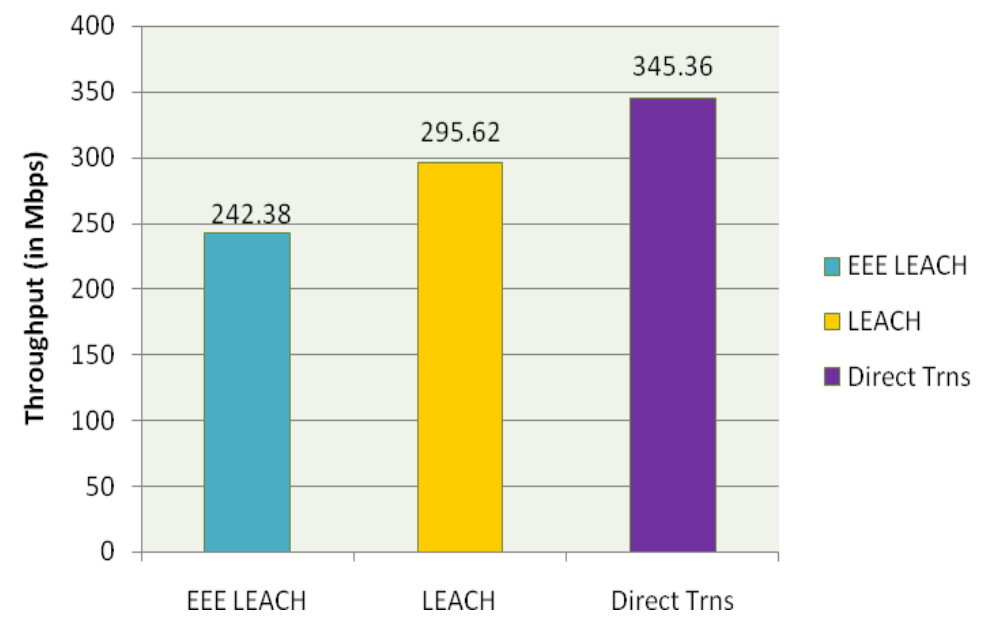

Figure 8: Throughput Graph Comparison between EEE LEACH, LEACH and DTx

Figure 8 depicts the comparison graph of the throughput values of the EEE LEACH, LEACH and DTx protocol. Larger the transmission time, smaller the throughput and it becomes clear from the formula used for throughput.

Throughput $=($ Size of the packet $/$ Transmission time $)$

Since EEE LEACH protocol takes larger time to transmit data to the BS, so the amount of data packets received by the BS per unit time is less than it is in LEACH and DTx protocol. Therefore EEE LEACH protocol shows smaller throughput than LEACH and DTx protocol.

\section{CONCLUSION}

In this paper we have discussed three routing protocols i.e. EEE LEACH, LEACH and DTx in Wireless Sensor Network and mainly focused on newly developed hierarchical routing protocol EEE LEACH. It was already proposed that EEE LEACH has greater energy efficiency with better network lifetime than LEACH protocol [2]. In this paper we tried to calculate it's transmission time and throughput and compared the results with LEACH and one another protocol i.e. DTx. Since EEE LEACH is more energy efficient and has shown better network lifetime, but here we have found that EEE LEACH takes more time to transmit data to BS which causes degradation of its throughput value. Therefore EEE LEACH protocol shows inefficient result in case of transmission time and throughput

\section{REFERENCES}

[1] Kalpana Sharma, Anurag S. Rathor, S. R. Biradar, M.K Ghose, "Power-efficient Routing \& Increased Yield Approach For WSNs," International Journal on Computer Science and Engineering Vol. 02, No. 03, 2010, 586-592. 
Advanced Computing: An International Journal ( ACIJ ), Vol.3, No.6, November 2012

[2] Meenakshi Sharma, Kalpana Sharma, "An Energy Efficient Extended LEACH (EEE LEACH)", 2012 International Conference on Communication Systems and Network Technologies, IEEE DOI 10.1109/ CSNT.2012.88, Rajkot, INDIA.

[3] Pedro de la Fuente Aragón, "The improvements of power management for clustered type large scope wireless sensor networks," Warsaw university of technology, June 2010.

[4] Wairagu G. Richard, "Extending LEACH routing algorithm for Wireless Sensor Network," Data Communications Engineering, Makerere University, 2009.

[5] Rajesh Patel, Sunil Priyani, Vijay Ukani, "Energy and Throughput Analysis of Hierarchical Routing Protocol (LEACH) for Wireless Sensor Network, "International Journal of Computer Applications (975 - 8887) Volume 20- No.4, April 2011.

[6] Nidhi Barta, Anuj Jain, Surender Dhiman, “An Optimized Energy Efficient Routing Algorithm for Wireless Sensor Network, International Journal of Innovative Technology and Creative Engineering (ISSS: 2045-8711) Vol 1 No.5 May 2011.

[7] C.Y. Chong and S.P. Kumar, "Sensor Networks: Evolution, opportunities, and Challenges", Proceedings of the IEEE, vol. 91, no. 8, Aug. 2003, pp. 1247-1256.

[8] Heinzelman, A. Chandrakasan and A. Balakrichnan, "Energy- Efficient Communication Protocol for Wireless Microsensor Networks", In proceedings of the 33rd Hawaii International Conference on System Science, Jan 2000.

[9] Kemal Akkaya and Mohamed Younis, “A Survey on Routing Protocols for Wireless Sensor Networks" Department of Computer Science and Electrical Engineering University of Maryland, Baltimore County Baltimore, MD 21250.

[10] Gizem, Meenakshi Sharma, Kalpana Sharma, "A Comparative Study of Hierarchical and QoS Based Routing Protocols in Wireless Sensor Network : A Survey", In Proceeding of "National Conference on Advance in Computer science \& information Security", PP 11-17, 6-7 January 2012, MIT, Manipal, India.

[11] Fan Xiangning, Song Yulin "Improvement on LEACH Protocol of Wireless Sensor Network", In Proceeding of "International Conference on Sensor Technologies and Applications", PP 260264, 2007

[12] Wu Xinhua, Wang Sheng, "Performance Comparison of LEACH and LEACH-C Protocols by NS2", In Proceeding of "Ninth International Symposium on Distributed Computing and Applications to Business, Engineering and Science”, PP 254-258, 2010.

[13] Yun Li, Nan Yu, Weiyi Zhang, Weiliang Zhao, Xiaohu You, Mahmoud Daneshmand, "Enhancing the Performance of LEACH Protocol in Wireless Sensor Networks", In Proceeding of “M2MCN-2011”, PP 223-228, 2011

[14] Jia Xu,Ning Jin, Xizhong Lou,Ting Peng,Qian Zhou,Yanmin Chen,“ Improvement of LEACH protocol for WSN", "International Conference on Fuzzy Systems and Knowledge Discovery (FSKD 2012)”, PP 2174-2177, 2012

[15] Yun Cosmin Cirstea, Mihail Cernaianu, and Aurel Gontean, "Packet Loss Analysis in Wireless Sensor Networks Routing Protocols”, “TSP-2012”, PP 37-41, 2012

\section{Authors}

Meenakshi Sharma has completed B.Tech degree in CSE department from North Eastern Regional Institute of Science \& Technology (NERIST), Arunachal Pradesh, INDIA in 2010. She has completed her M.Tech degree in CSE department from Sikkim Manipal Institute of Technology (SMIT), Majhitar, East Sikkim, INDIA in the year of 2012 .

Anil Kumar Shaw has completed B.Tech degree in ECE department from North Eastern Regional Institute of Science and Technology (NERIST), Arunachal Pradesh, INDIA in 2010. He is currently perusing his M.Tech in ECE department from NERIST, Arunachal Pradesh, INDIA,

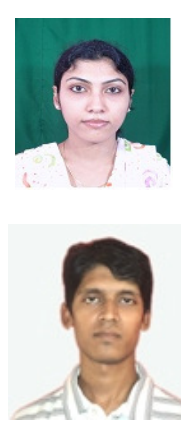

